\title{
Casos Clínicos \\ La tos crónica intratable no siempre es asma
}

\author{
Chronic intractable cough is not always asthma
}

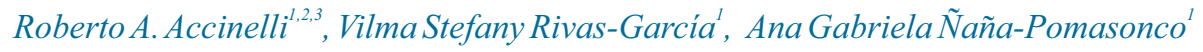

La capital del Perú es la segunda ciudad más poblada del mundo en medio de un desierto. Por 8 a 9 meses por año está cubierta por un manto de nubes producto de la presencia de la cordillera de los Andes y de la corriente de Humboldt. Por esta inversión térmica en Lima la prevalencia de asma es muy elevada, ocupando el primer lugar de frecuencia en respuesta al cuestionario visual del estudio ISAAC ${ }^{(1)}$. Por ello la tendencia es a considerar a cualquier persona que se queje de tos perenne y ronquera de pecho como portador de asma, existiendo otras enfermedades que pueden explicar los síntomas que presente el paciente. Presentamos el caso de una adulta mayor vista por tres años por diferentes médicos, habiendo incluso sido hospitalizada por esta enfermedad que finalmente no lo fue.

\section{Caso clínico}

Mujer de 57 años quien consultó por dolor torácico a predominio de la base derecha, malestar general e intensa tos intratable. Había salido de alta sesenta días atrás de otra institución después de estar internada durante una semana por asma.

Funciones biológicas: Sueño disminuido por tos. Ánimo deprimido.

Al examen se encontró a una paciente en buen estado general con disfonía que lucía deprimida.

\section{Antecedentes:}

Fumaba ocasionalmente.

Tres años atrás por 20 días de ronquera de pecho, "flemas pegadas", congestión nasal, disnea de esfuerzo y opresión torácica fue diagnosticada de asma bronquial, siendo internada por una semana. Su saturación de oxígeno fue $96 \%$, su frecuencia respiratoria de 22 por minuto y en ambos campos pulmonares presentaba roncantes y sibilantes. Dos años después fue hospitalizada por una segunda, y hace alrededor de 8 meses atrás por una tercera vez, en que le hicieron una espirometría cronometrada con y sin broncodilatadores que fue completamente normal (Tabla 1)

Hace siete meses acudió a un otorrinolaringólogo quien le diagnosticó hipertrofia de los cornetes nasales. Tres meses después acudió nuevamente a él por cefalea y sensación de obstrucción de los oídos. La tomografía espiral multicorte (TEM) de senos paranasales no mostró sinusitis (Figura 1). En la laringofibroscopía se evidenció hongos en la laringe. Un mes después recibió tratamiento con nistatina.

Por incremento del compromiso bronquial, cansancio generalizado y ronquera, tuvo su cuarto internamiento recibiendo broncodilatadores, corticoides endovenosos e inhalados, levofloxacina. Tampoco requirió oxígeno ni le realizaron una espirometría. Luego de 9 días fue dada de alta con prednisona $50 \mathrm{mg}$ por 3 días, fluticasona/salmeterol $25 / 125$ $\mu \mathrm{g} 2$ puff cada 12 horas, tiotropio $2,5 \mu \mathrm{g} 2$ inhalaciones por noche, salbutamol $100 \mu \mathrm{g} 2$ puff cada 6 horas. Le tomaron una radiografía (Figura 2) y una TEM (Figura 3) de tórax La paciente consideraba que "salió igual a como entró", por lo que a los tres días del alta acudió ver a su neumólogo, quien la nebulizó. A la semana siguiente acudió nuevamente al otorrinolaringólogo por congestión nasal, inflamación de garganta, afonía, obstrucción de oídos quien le indicó levofloxacino $500 \mathrm{mg}$ una tableta por siete días, deflazacort 6 $\mathrm{mg}$ dos tabletas diarias por cinco días, cetirizina/pseudoefedrina 100/600 mg dos tabletas diarias por cinco días; además de enjuague con bicarbonato de sodio con el que sentía mejoría. Le recomendaron que se operara los cornetes porque le estaban causando la obstrucción de las vías respiratorias.

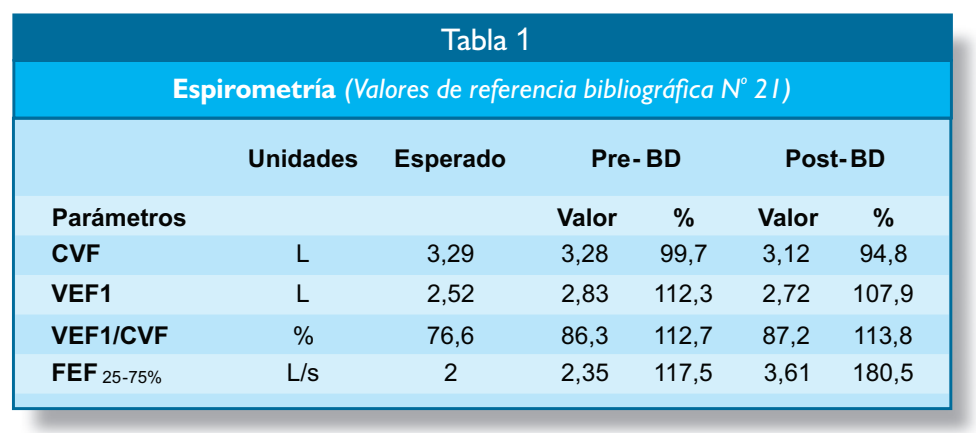

${ }^{1}$ Instituto de Investigaciones de la Atura. Universidad Peruana Cayetano Heredia. ${ }^{2}$ Facultad de Medicina Alberto Hurtado. Universidad Peruana Cayetano Heredia. ${ }^{3}$ Hospital Cayetano Heredia 


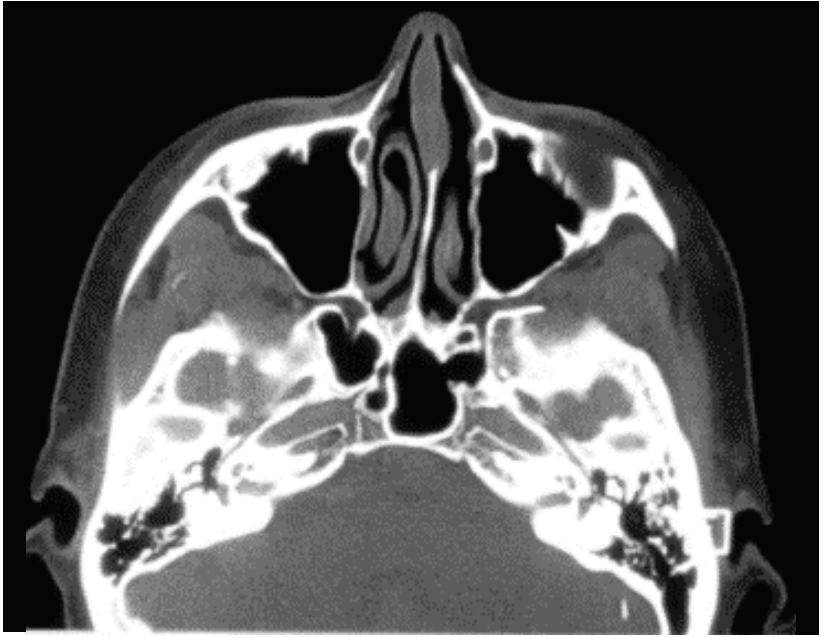

Figura 1. Tomografía de los senos paranasales en que se muestra su interior completamente libre.

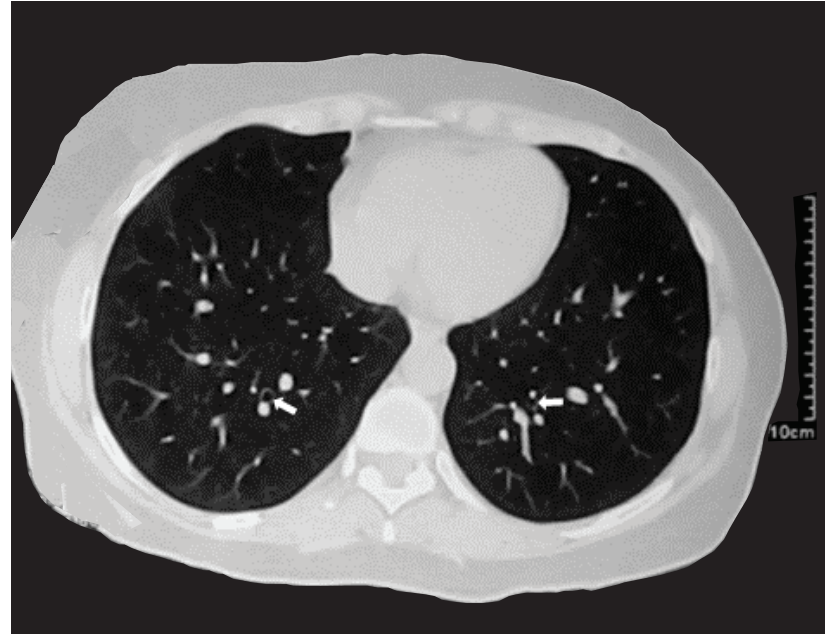

Figura 3. En el corte transversal de la tomografía a nivel de las bases se observa que los bronquios cortados a través son de mayor diámetro que el vaso que acompaña, signo de bronquiectasia.

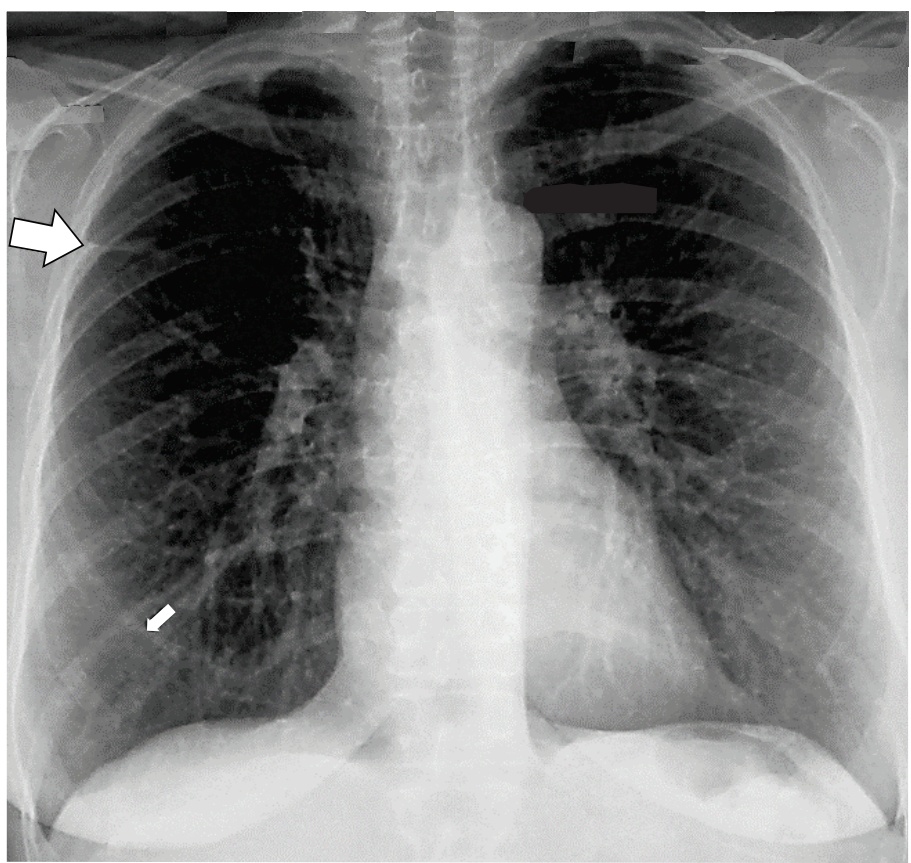

Figura 2. Radiografía de tórax en incidencia póstero-anterior que muestra 10 espacios intercostales. Se observa un engrosamiento de la pleura apical que sigue hasta la cisura mayor (Flecha blanca gruesa). Hay calcificaciones periféricas de los cartílagos condrocostales, observándose mejor en el sexto derecho (Flecha blanca delgada).

La paciente desde más de un año atrás diariamente al levantarse tenía su voz más gruesa, lo que lo atribuía a "hablar demasiado", por lo que recibió terapia de voz y gárgaras de bicarbonato. Negaba los síntomas clásicos de reflujo gastro esofágico como son ardor retro-esternal y sensación de subida de los alimentos hasta la boca, pero refería que cuando tomaba gaseosas sí los presentaba. Con el café, pues tomaba 3 tazas diarias, con las gaseosas, que consumía 3 veces al día, con los cítricos y con las comidas que contienen albahaca presentaba tos.

\section{Evolución:}

Diagnosticada de tos intratable por enfermedad por reflujo gastroesofágico, se suspendió el tratamiento que recibía. Con medidas antireflujo, y terapia farmacológica para su ansiedad con clonazepam y para la depresión con amitriptilina a la semana ya no tenía tos pero sí intermitentemente disfonía. Su espirometría fue normal (Tabla 2). Tres semanas después refería estar normal, sin ningún tratamiento para el asma. 


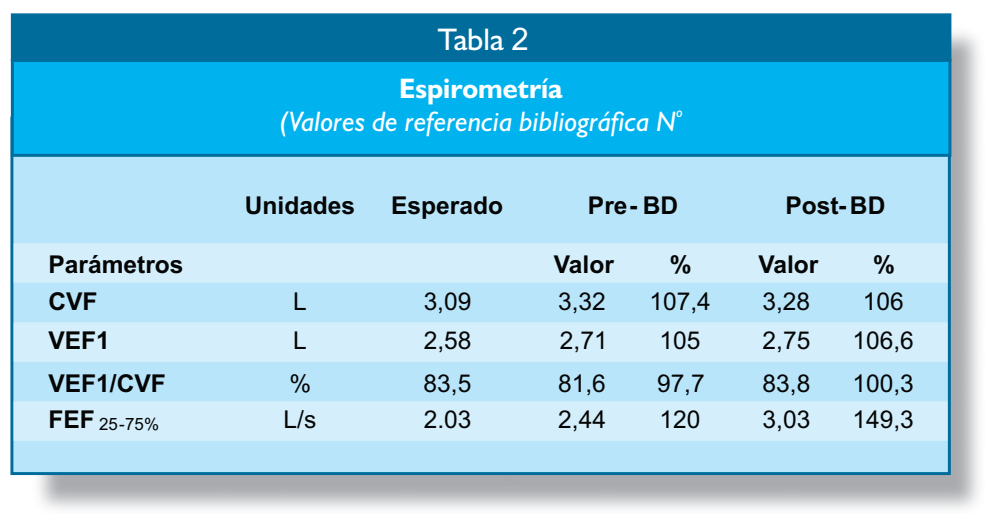

\section{Discusión}

La paciente había sido hospitalizada por asma no controlada pero nunca necesitó recibir oxígeno. Los pacientes que acuden a de emergencia con asma aguda presentan dificultad para respirar, opresión en el pecho, tos y/o sibilancias ${ }^{(2)}$, y si no responden al tratamiento terminan hospitalizados. La paciente podría haber tenido asma de difícil manejo, en la que los valores de sus estudios de función pulmonar estarían por debajo de los normales ${ }^{(3)}$, pero esta paciente tuvo dos espirometrías con valores por encima del $100 \%$ (Tablas 1 y 2 ). Factore asociados a pobre control incluyen el ingreso hospitalario previo por asma, y ella nunca había sido internada por esta enfermedad previamente, y que en los cuestionarios de control de asma se encuentren valores elevados, los que nunca se le realizaron ${ }^{(4)}$.

Esta paciente no tuvo criterios para ser internada por asma. Y es por ello que aunque el tratamiento que se le dio para el asma fue adecuado no sintió ninguna mejoría. Pero el médico que la vio al tercer día de su alta al estar igual optó por volver a nebulizarla. Posteriormente le plantearon que debía ser operada pues lo que tenía mejoraría si se le operaban los cornetes. Pero la TEM de senos paranasales no muestra una obstrucción que amerite cirugía ni al examen clínico se encontró que tuviera obstruidas las fosas nasales.

$\mathrm{Su}$ TEM de pulmones no muestra alteraciones parenquimales y el único hallazgo es que en las bases se encuentra una relación del diámetro del bronquio con la arteria adyacente mayor a uno (Figura 2), lo que hace el diagnóstico de bronquiectasias ${ }^{(5)}$. Salvo ese hallazgo su radiografía de tórax es normal (Figura 3).

Estamos entonces ante una paciente con tos crónica con radiografía de tórax normal. Como la paciente no usaba inhibidores de la enzima convertidora de la angiotensina ${ }^{(6)}$ los otros diagnósticos posibles son tres: Asma bronquial, rinitis y enfermedad por reflujo gastroesofágica $(\mathrm{ERGE})^{(7)}$. Hemos visto que asma no presentaba y rinitis tampoco.

Analicemos la tercera posibilidad. ERGE se puede presentar sin los síntomas clásicos, como es la acidez retroesternal, forma a la que se le llama ERGE silenciosa ${ }^{(8)}$. Esta paciente se quejaba que desde un año atrás había notado que al levantarse su voz era más gruesa, y de presentar síntomas de RGE cuando tomaba café, gaseosas y alimentos con albahaca. Estas bebidas y comidas se han relacionado con $\mathrm{ERGE}^{(9)}$.
La tos en ERGE ha sido relacionada a un reflejo asociado a la presencia del contenido ácido del estómago en el esófago ${ }^{(10)}$. El otro mecanismo, que es el que esta paciente probablemente tendría, es que el ácido sube hasta las cuerdas vocales inflamándolas, lo que se traduce en disfonía, y llega hasta la tráquea y bronquios causando una marcada inflamación de las vías aéreas que explica la tos intensa que presenta esta paciente ${ }^{(11)}$. El tratamiento en estos casos es con cambio del estilo de vida ${ }^{(12)}$, pues el empleo de antiácidos, como los inhibidores de la bomba de protones, solo haría más alcalino el reflujo. Es por ello que en los múltiples estudios que se han realizado en pacientes con asma y ERGE con tratamiento solo con antiácidos no han mostrado ser efectivos ${ }^{(13,14)}$.

La paciente fue sometida a una estricta dieta eliminando todos los alimentos y bebidas que pueden exacerbar la ERGE y se le indicó además las medidas de cambio en su estilo de vida con lo que en una semana la tos desapareció, para después de tres semanas sentirse normal, a pesar de haberse suspendido simultáneamente todo el tratamiento del asma.

En la enfermedad por reflujo, los síntomas son malos marcadores de la gravedad de la enfermedad. Las estimaciones epidemiológicas basadas en pacientes referidos para endoscopia casi seguramente sobreestimarán la verdadera prevalencia de la enfermedad de reflujo erosivo en la población ${ }^{(15)}$. Para evitar sesgos, la forma más adecuada de conocer la prevalencia de ERGE y de esofagitis erosiva sería por un estudio poblacional en que se hiciera una encuesta buscando sus síntomas y se realizaran endoscopías digestivas altas a todas las personas encuestadas, así fueran asintomáticas. En el estudio Kalixanda se tomó una muestra aleatoria de 3000 personas hallando una prevalencia de síntomas de ERGE de $40,0 \%$, y entre 1000, también seleccionadas al azar, por esófago-gastro-duodeno endoscopía se encontró que 15,5\% tenía esofagitis erosiva (EE). De los que tenían ERGE sintomática el 24,5\% tenía EE, mientras que el 36,8\% de los que tenían EE no tenía síntomas de $\mathrm{ERGE}^{(16)}$. Estos resultados explican la dificultad del diagnóstico de ERGE, el cual debe ser planteado en las personas como la paciente que describimos, con síntomas de este problema digestivo, pero además con radiografía de tórax normal y tos crónica que no respondía a los tratamientos prescritos para asma y rinitis.

En la enfermedad por reflujo, los síntomas son malos marcadores de la gravedad de la enfermedad. Las estimaciones epidemiológicas basadas en pacientes referidos para endoscopía casi seguramente sobreestimarán la verdadera 
prevalencia de la enfermedad de reflujo erosivo en la población (15). Para evitar sesgos, la forma más adecuada de conocer la prevalencia de ERGE y de esofagitis erosiva sería por un estudio poblacional en que se hiciera una encuesta buscando sus síntomas y se realizaran endoscopías digestivas altas a todas las personas encuestadas, así fueran asintomáticas. En el estudio Kalixanda se tomó una muestra aleatoria de 3000 personas hallando una prevalencia de síntomas de ERGE de $40,0 \%$, y entre 1000 , también seleccionadas al azar, por esófago-gastro-duodeno endoscopía se encontró que 15,5\% tenía esofagitis erosiva (EE). De los que tenían ERGE sintomática el $24,5 \%$ tenía EE, mientras que el 36,8\% de los que tenían EE no tenía síntomas de ERGE ${ }^{(16)}$. Estos resultados explican la dificultad del diagnóstico de ERGE, el cual debe ser planteado en las personas como la paciente que describimos, con síntomas de este problema digestivo, pero además con radiografía de tórax normal y tos crónica que no respondía a los tratamientos prescritos para asma y rinitis.

\section{Referencias bibliograficas}

1. The International Study of Asthma and Allergies in Childhood (ISAAC) Steering Committee. Worldwide variation in prevalence of symptoms of asthma, allergic rhinoconjunctivitis, and atopic eczema: ISAAC. Lancet. 1998;351(9111):1225-32.

2. Fergeson JE, Patel SS, Lockey RF. Acute asthma, prognosis, and treatment. J Allergy Clin Immunol. 2017;139(2):438-47.

3.Schatz M, Zeiger RS, Vollmer WM, Mosen D, Cook EF. Determinants of future long-term asthma control. J Allergy Clin Immunol. 2006;118(5):1048-53.

4. Patel M, Pilcher J ,Reddel HK, Qi V, Mackey B, Tranquilino T, Shaw D, Black P, Weatherall M, Beasley R; SMART Study Group. Predictors of severe exacerbations, poor asthma control, and $\beta$-agonist overuse for patients with asthma. J Allergy Clin Immunol Pract. 2014;2(6):751-58.

5. Kimmig L, Bueno J. Dilated Bronchi: How Can I Tell? Ann Am Thorac Soc. 2017;14(5):807-9.

6. Mu G, Xiang Q, Zhou S, Xie Q, Liu Z, Zhang Z, Cui Y. Association between genetic polymorphisms and angiotensinconverting enzyme inhibitor-induced cough: a systematic review and meta-analysis. Pharmacogenomics. 2019;20(3):189-212.

7. Turner RD, Bothamley GH. Chronic cough and a normal chest X-ray - a simple systematic approach to exclude common causes before referral to secondary care: a retrospective cohort study. NPJ Prim Care Respir Med. 2016;26:15081.

8. Kim SE. Clinical Considerations of Silent Gastroesophageal Reflux Disease in Morbidly Obese Patients. Gut Liver. 2017;11(3):315-16.

9. Caselli M, Lo Cascio N, Rabitti S, Eusebi LH, Zeni E, Soavi C, Cassol F, Zuliani G, Zagari RM. Pattern of food intolerance in patients with gastro-esophageal reflux symptoms. Minerva Med. 2017;108(6):496-501.

10. Lee JH, Park SY, Cho SB, Lee WS, Park CH, Koh YI, Joo YE, Kim HS, Choi SK, Rew JS. Reflux episode reaching the proximal esophagus are associated with chronic cough. Gut Liver. 2012; 6(2):197-202.

11. Crausaz FM, Favez G. Aspiration of solid food particles info lungs of patients with gastroesophageal reflux and chronic bronchial disease. Chest 1988;93:376-78.

12. Haruma K, Kinoshita Y, Sakamoto S, Sanada K, Hiroi S, Miwa H. Lifestyle factors and efficacy of lifestyle interventions in gastroesophageal reflux disease patients with functional dyspepsia: primary care perspectives from the LEGEND study. Intern Med. 2015;54(7):695-701.

13. Riscili BP, Parsons JP, Mastronarde JG. Treating silent reflux disease does not improve poorly controlled asthma.Cleve Clin J Med. 2010;77(3):155-60.

14. Sopo SM, Radzik D, Calvani M. Does treatment with proton pump inhibitors for gastroesophageal reflux disease (GERD) improve asthma symptoms in children with asthma and GERD? A systematic review. J Investig Allergol Clin Immunol. 2009;19(1):1-5.

15. Bytzer P. Gastro-oesophageal reflux disease: Epidemiologic challenges. Scandinavian Journal of Gastroenterology, 2005;40:247-49.

16. Ronkainen J, Aro P, Storskrubb T, Johansson SE, Lind T, Bolling-Sternevald E, et al. High prevalence of gastroesophageal reflux symptoms and esophagitis with or without symptoms in the general adult Swedish population: a Kalixanda study report. Scand J Gastroenterol 2005;40:27585 .

Citar como: Accinelli RA. Rivas-García VS. Ñaña-Pomasonco AG. La tos crónica intratable no siempre es asma. Diagnóstico(Lima).2019;58(2):101-4. DOI: https://doi.org/10.33734/diagnostico.v58i2.214

Correspondencia: Roberto A. Accinelli Dirección: Av. Honorio Delgado 430 - San Martín de Porres 15102 -Lima Teléfono: 998119480

Correo electrónico: roberto.accinelli@upch.pe 